\title{
Help-Seeking for Insomnia among Adult Patients in Primary Care
}

\author{
James E. Aikens, PhD, and Mary E. Rouse, PhD, MBChB, MRCGP
}

Objective: To clarify the patient characteristics associated with seeking medical help for insomnia.

Methods: A consecutive sample $(n=700)$ of adults attending a nonurgent primary care appointment was screened for sleep problems. A follow-up mailed survey then assessed insomnia symptoms, daytime impairment, beliefs about sleep, medication use, sleepiness and fatigue, and medical help-seeking.

Results: Fifty-two percent of patients with probable insomnia reported discussing this with a physician. Multivariate logistic regression analyses indicated that discussing one's probable insomnia with a physician was independently associated with having a greater number of medical conditions (OR, 2.19 [95\% CI, 1.13 to 4.22]), being more highly educated (1.67 [95\% CI, 1.11 to 2.51]), sleeping less per night (OR, 0.71 [95\% CI, 0.52 to 0.96$]$ ), and greater perceived daytime impairment due to insomnia (OR, 2.07 [95\% CI, 1.06 to 4.03]).

Conclusions: Primary care patients often seek medical help when they experience insomnia. Clinical treatment ought to especially target poor sleepers presenting with significant insomnia-related impairment, medical morbidity, or both. Future research should further clarify the role of medical and psychiatric comorbidities and determine whether resolution of insomnia and its consequences improves the outcomes of common medical conditions. (J Am Board Fam Pract 2005;18:257-61.)

Approximately $40 \%$ of community-dwelling adults and $69 \%$ of primary care patients report sleep problems in the form of difficulty falling asleep, discontinuous sleep, and/or nonrestorative sleep. ${ }^{1-3}$ Insomnia is associated with motor vehicle accidents, ${ }^{4}$ psychiatric disorders, ${ }^{2,5,6}$ impaired role functioning, ${ }^{2}$ cognitive difficulties, ${ }^{7}$ and increased medical utilization. Although effective behavioral and pharmacologic therapies exist for insomnia, ${ }^{8,9}$ only $31 \%$ of poor sleepers discuss their insomnia with a physician. ${ }^{3}$ Experts in sleep medicine have accordingly called for improved insomnia recognition and treatment. ${ }^{10-12}$

However, consideration of the patient's perspective suggests that the problem is not simply physician failure to recognize sleep problems; many patients with syndromally defined insomnia do not view their sleep problem as clinically significant. Of the $9 \%$ of American adults reporting the most severe sleep loss, one third do not consider their

Submitted, revised, 28 February 2005.

From the Department of Family Medicine (JEA) and Psychiatry (JEA), University of Michigan Medical School, Ann Arbor, MI; and Glover Street Medical Centre (MER), Perth, UK.

Conflict of interest: none declared.

Corresponding author: Dr. J. E. Aikens, Department of Family Medicine, University of Michigan, 1018 Fuller Street (Box 0708), Ann Arbor, MI 48109 (e-mail: aikensj@umich.edu). problem to be serious, ${ }^{12}$ and only $24 \%$ of British adults who report insomnia in surveys also describe themselves as dissatisfied about their sleep. ${ }^{13}$ Thus, more information is needed to understand the patient-centered factors involved in insomnia presentation. This information may help explain why only some poor sleepers seek help, identify subgroups of poor sleepers to target for intensive intervention efforts, and identify intervention targets (beyond sleep quality) of primary importance to patients.

We conducted this study in an attempt to identify the specific aspects of insomnia that motivate syndromally defined problem sleepers to seek medical help. Although a prior study ${ }^{3}$ found that insomnia help-seeking was partially explained by ratings of "how you feel physically," this nonspecific measure of somatic distress is difficult to interpret. Because general somatic distress might rise from daytime sleepiness or daytime fatigue, comorbid medical illness or pain, or other issues, we also assessed medical morbidity and several distinct aspects of daytime functioning (sleepiness, cognitive and emotional fatigue, and impairment attributed to poor sleep).

\section{Methods \\ Procedure and Measures}

Subjects were recruited consecutively from a series of adults ( $\geq 18$ years old) attending nonurgent fam- 
ily practice appointments at either a 12-physician suburban-based practice primarily serving University personnel and their families or a 9-physician urban-based family practice primarily serving an ethnically diverse lower-middle income population. Patients were solicited after arrival registration, using staff-distributed study flyers and face-to-face invitation by research assistants. Recruitment attempts were distributed across 6 days of the week and across mornings and afternoons.

Consenting patients received $\$ 2$ for completing a 2-minute written screening survey that covered demographics and medical characteristics and included the Insomnia Severity Index (ISI). ${ }^{14}$ Based on DSM-IV insomnia criteria, ${ }^{15}$ the ISI elicits 0 to 4 severity ratings ("none" to "very"/"very much") of recent problems with sleep onset, sleep maintenance, and early morning awakening, interference with daytime functioning, perceived conspicuousness of impairment, concern about sleep problems, and satisfaction with sleep patterns. A composite score is obtained by summing ratings; higher scores indicate more severe insomnia. Good internal consistency test-retest stability and evidence of validity have been reported. ${ }^{16}$ Per scoring guidelines, we applied a cutoff total of $\geq 8$ to indicate probable insomnia.

Within 1 week and usually within 24 hours of the waiting room screening, subjects with ISI-defined problem sleep were mailed a self-report packet to further assess sleep disturbance, daytime functional impairment, the use of sleep strategies, and discussion of the problem with a physician. Detailed sleep information was gathered with the 19-item Pittsburgh Sleep Quality Index (PSQI), ${ }^{17}$ which yields a reliable and stable total score that is 90\% sensitive and $87 \%$ specific for probable insomnia, as well as subscales reflecting subjective sleep quality, sleep latency, sleep duration, habitual sleep efficiency, sleep disturbances, medication use, and daytime dysfunction. Beliefs about sleep were measured with the Dysfunctional Beliefs About Sleep (DBAS) scale, ${ }^{18}$ which assesses misconceptions about insomnia causes, misattributions about its consequences, excessive expectations of sleep amount, control over and predictability of sleep, and erroneous beliefs about insomnia remediation. The Epworth Sleepiness Scale (ESS) ${ }^{19}$ was used to assess propensity for unintended dozing in several daytime situations; this widely used 8-item instrument has acceptable validity, test-retest reliability, and internal consistency. ${ }^{20}$ Daytime fatigue was assessed using the Modified Fatigue Impact Scale (MFIS), ${ }^{21}$ which has 21 items assessing overall fatigue as well as its effects on physical, cognitive, and psychosocial functioning. A cover letter requested that subjects return the instrument using a postagepaid return envelope, and one reminder telephone call was made to encourage completion. Those who returned the mailed questionnaire were paid an additional $\$ 10$. Both protocol phases were Institutional Review Board approved.

\section{Statistical Analysis}

Descriptive statistics were computed, and variables with non-normal distributions were rank-ordered or log-transformed for analysis. Missing data points were not imputed. Bivariate associations were evaluated using $\chi^{2}$ statistics for categorical associations, Student $t$ tests for categorical by continuous associations, and Pearson correlations for continuous associations. Correlation matrices were examined to eliminate from the model any major variables not having a significant zero-order correlation with the outcome variables. A multivariate logistic regression model was estimated using forward selection procedures $(P($ enter $)<.05, P($ remove $) \geq .10)$.

\section{Results}

Of 700 patients solicited, 632 (90\%) consented to undergo initial screening, 326 (52\%) of whom scored positive on one or both of the screening instruments and were mailed a survey. Return rate was $55 \%$. Survey return (Table 1) was unrelated to age and ISI total scores but was less likely among subjects who were either male $(P=.03)$ or of racial/ethnic minority $(P<.001)$.

Of the 180 subjects who returned the survey, $72 \%$ met criteria for probable insomnia, but $12 \%$ had incomplete surveys. The resulting 115 "prob-

Table 1. Comparison of Mailed Survey Responders and Nonresponders

\begin{tabular}{lccr}
\hline Variable & $\begin{array}{c}\text { Responders } \\
(\mathrm{n}=180)\end{array}$ & $\begin{array}{c}\text { Nonresponders } \\
(\mathrm{n}=146)\end{array}$ & $P$ \\
\hline Age & $40.4 \pm 14.2$ & $39.2 \pm 12.5$ & .442 \\
Gender (female) & $71.0 \%$ & $59.5 \%$ & .030 \\
Some college education & $68.9 \%$ & $63.4 \%$ & .289 \\
Racial/ethnic minority & $16.0 \%$ & $35.6 \%$ & $<.001$ \\
Insomnia Severity Index & $14.4 \pm 4.5$ & $14.1 \pm 4.6$ & .493 \\
\hline
\end{tabular}


Table 2. Characteristics of Probable Insomnia Sample

\begin{tabular}{|c|c|}
\hline & $\begin{array}{c}\text { Mean } \pm \mathrm{SD} \text { or } \\
\text { Percentage }\end{array}$ \\
\hline Age & $39.0 \pm 13.4$ \\
\hline Gender (female) & $70 \%$ \\
\hline $\begin{array}{l}\text { Ethnic/racial status } \\
\text { White } \\
\text { African American } \\
\text { Other }\end{array}$ & $\begin{array}{r}85 \% \\
13 \% \\
2 \%\end{array}$ \\
\hline $\begin{array}{l}\text { Level of education } \\
\text { No high school degree } \\
\text { High school degree } \\
\text { Some college } \\
\text { College degree } \\
\text { Advanced degree }\end{array}$ & $\begin{array}{r}6 \% \\
24 \% \\
39 \% \\
18 \% \\
12 \%\end{array}$ \\
\hline $\begin{array}{l}\text { Number of medical conditions } \\
0 \\
\quad 1 \text { to } 2 \\
\geq 3\end{array}$ & $\begin{array}{l}11 \% \\
45 \% \\
44 \%\end{array}$ \\
\hline $\begin{array}{l}\text { Self-rated health status } \\
\text { Excellent } \\
\text { Very good } \\
\text { Good } \\
\text { Fair } \\
\text { Poor }\end{array}$ & $\begin{array}{r}9 \% \\
26 \% \\
45 \% \\
15 \% \\
5 \%\end{array}$ \\
\hline $\begin{array}{l}\text { Sleep features } \\
\text { Duration of problem sleep } \\
<1 \text { month } \\
1 \text { to } 3 \text { months } \\
4 \text { to } 6 \text { months } \\
6 \text { to } 12 \text { months } \\
>12 \text { months } \\
\text { Usual sleep duration per night (in hours) } \\
\text { Insomnia Severity Index } \\
\text { Discussed sleep problem with physician }\end{array}$ & $\begin{array}{c}2 \% \\
9 \% \\
9 \% \\
9 \% \\
71 \% \\
5.7 \pm 1.6 \\
15.5 \pm 4.5 \\
52 \%\end{array}$ \\
\hline
\end{tabular}

able insomnia" cases (Table 2) had an average age of 39 years. Seventy percent were women, $15 \%$ were of racial or ethnic minority, $89 \%$ had at least one medical condition, and $70 \%$ had at least some college education. The mean ISI score (15.5) indicates clinical insomnia of moderate severity.

Preliminary analyses were used to identify variables with significant bivariate associations with help-seeking. Compared with those who did not discuss their sleep problem $(n=60,52 \%)$ with a physician, those who did discuss it $(\mathrm{n}=55)$ had significantly more comorbid conditions $(P=.016)$ and rated their overall health more poorly $(P=$ .030). However, there were no differences in age, gender, or ethnicity (all $P$ values $>.817$ ), although there was a possible trend suggesting higher levels of education among patients with a history of having discussed their problem $(P=.074)$. In terms of sleep variables, help-seekers reported significantly greater difficulty with sleep maintenance $(P=.011)$ but not with sleep onset $(P=.266)$ or early morn-
Table 3. Results of Multivariate Logistic Regression

\begin{tabular}{lrcc}
\hline Predictor & Unst. $\beta$ & OR $(95 \%$ CI $)$ & $P$ \\
\hline $\begin{array}{l}\text { Number of comorbid } \\
\quad \text { conditions* }\end{array}$ & 0.78 & $2.19(1.13$ to 4.22$)$ & .020 \\
$\begin{array}{l}\text { Level of education } \\
\text { Mean sleep duration per } \\
\text { night (past month) }\end{array}$ & -0.35 & $0.71(0.52$ to 0.96$)$ & .026 \\
$\begin{array}{l}\text { Insomnia-related daytime } \\
\text { impairment }\end{array}$ & 0.73 & $2.07(1.06$ to 4.03$)$ & .033 \\
\hline
\end{tabular}

* On a scale of 0,1 to 2 , or $3+$ conditions.

${ }^{\dagger}$ On a 5 -point scale, as detailed in Table 2.

† On a 3-point scale ('somewhat,' 'much,' 'very much'; subjects who responding less than 'somewhat' did not meet sample criteria).

ing awakening $(P=.408)$. They also reported significantly less time asleep per night (mean difference, 56 minutes, $P=.002$ ), poorer overall sleep quality $(P<.050)$, significantly greater interference of their poor sleep with their daytime activities $(P=.009)$, and greater overall dissatisfaction with their sleep $(P=.019)$. There were no significant differences in daytime sleepiness, duration of problem sleep, or mental health index (all $P$ values $>.520$ ), although there was a possible trend suggesting greater daytime fatigue among patients with a history of discussing sleep with a physician $(P=.078)$.

Finally, we conducted binomial regression modeling to identify the best predictors of help-seeking. The final model (Table 3) included 4 variables. Help-seeking became more likely among patients with a greater number of medical conditions (OR, 2.19; $95 \%$ CI, 1.13 to 4.22 ), better-educated patients (OR, 1.67; 95\% CI, 1.11 to 2.51 ), patients who reported less total sleep time per night (OR, $0.71 ; 95 \% \mathrm{CI}, .52$ to .96$)$, and patients who reported greater daytime impairment due to insomnia (OR, 2.07; 95\% CI, 1.06 to 4.03 ).

\section{Discussion}

Findings indicated that help-seeking for problem sleep was most likely among patients with multiple medical conditions who have more severe insomnia and believe that the sleep problem affects daytime functioning. In contrast, another recent survey by Shocat et $\mathrm{al}^{3}$ found that help-seeking for sleep problems was related to younger age, higher income level, a longer insomnia course, and "feeling poor physically" during the daytime. Our differing results may be due to our quantification of medical 
morbidity, methodological differences in the measurement of chronicity and daytime function, sample size, and/or geographic variation.

Even after accounting for insomnia severity, daytime consequences remained associated with help-seeking. That is, regardless of insomnia severity, patients seem especially motivated to resolve their insomnia when they view it as intruding on their role performance or other goals. This finding implies that case-finding efforts ought to especially focus on identifying insomnia accompanied by significant daytime consequences. Moreover, physicians who treat insomnia ought to focus beyond sleep improvement per se, because some patients may be satisfied by an improved functional outcome even if their sleep is not perfectly normalized by treatment.

The association between help-seeking and medical comorbidity could be due to at least 2 mechanisms. First, sleep is disrupted by numerous medical conditions (eg, chronic and acute pain conditions, obstructive sleep apnea, gastric reflux disease, and thyroid imbalance, as well as by numerous medications). Treating physicians should remain aware that, although chronic disease will often seem to overshadow insomnia in importance, the latter is often easily controlled. Moreover, it seems likely that patients who sleep better will experience less overall distress and play a more active role in their disease management. Thus, adjunctive treatment of insomnia is a potentially efficient way for physicians to improve health-related quality-of-life among the chronically ill. A second possibility is that increased insomnia reporting among those with medical conditions is an artifact of increased physician contact as a result of care for the chronic disease itself. Although we did not collect medical utilization data, follow-up research might test the role of visit frequency in this association. Another interesting task for future work would be to compare levels of role impairment between patients with self-defined insomnia and those with other disabling conditions.

Certain study limitations should be noted. Foremost of these is that the cross-sectional data cannot support causal interpretations, making it impossible to disentangle the various possible mechanisms involved with medical morbidity. Second, despite our attempt to include reliable, well validated measures, poor recall and self-report biases may have led to inaccurate estimates, especially for issues such as sleep characteristics ${ }^{22}$ and perceived impairment. Response rate may have been reduced by assessment burden, in that the mailed survey required approximately 15 to 20 minutes to complete. Incorporation of reminder prompts or telephone interviews would probably improve response rate. The results might not validly extend to men and patients of ethnic minority origin, because of lower return rates among these subgroups. The lack of sleep disorder diagnostic assessments is another drawback, because occult problems such as obstructive sleep apnea could be the primary cause of insomnia for some subjects. However, this limitation is partly mitigated by the fact that our objective was to focus on symptom reporting irrespective of cause.

In closing, we conclude that patients with insomnia are slightly more likely to seek medical help for sleep than to not seek help for sleep. Because perceived daytime impairment seems to be an independent motivator of help-seeking, primary care physicians are advised to focus beyond sleep quality and amount per se and address patient concerns about the impact of poor sleep on their daytime functioning. Insomnia resolution should also be made a priority among patients with chronic illness. Future research should include standardized diagnostic interviews, which would provide a basis for testing the role of psychiatric symptoms and syndromes. Future work should also aim to determine whether outcomes are improved when the treatment focus is extended to include daytime consequences.

\section{References}

1. Roth T, Ancoli-Israel S. Daytime consequences and correlates of insomnia in the United States: results of the 1991 National Sleep Foundation Survey. II. Sleep 1999;22 Suppl 2:S354-8.

2. Simon GE, Von Korff M. Prevalence, burden, and treatment of insomnia in primary care. Am J Psychiatry $1997 ; 154: 1417-23$.

3. Shochat T, Umphress J, Israel AG, Ancoli-Israel S. Insomnia in primary care patients. Sleep 1999;22: S359-65.

4. The Gallup Organization. Sleep in America: a national survey of US adults. Los Angeles: National Sleep Foundation; 1991.

5. Aikens JE, Mendelson WB, Baehr EK. Replicability of psychometric differences between obstructive sleep apnea, primary snoring, and periodic limb movement disorder. J Sleep Hypnosis 2000;4:211-5.

6. Aikens JE, Vanable PA, Tadimeti L, Caruana- 
Montaldo B, Mendelson WB. Differential rates of psychopathological symptoms in periodic limb movement disorder, obstructive sleep apnea, psychophysiological insomnia, and insomnia with psychiatric disorder. Sleep 1999;22:775-80.

7. Zammit GK, Weiner J, Damato N, Sillup GP, McMillan CA. Quality of life in people with insomnia. Sleep 1999;22 Suppl 2:S379-85.

8. Morin CM, Hauri PJ, Espie CA, Spielman AJ, Buysse DJ, Bootzin RR. Nonpharmacologic treatment of chronic insomnia. An American Academy of Sleep Medicine review. Sleep 1999;22:1134-56.

9. Nowell PD, Mazumdar S, Buysse DJ, Dew MA, Reynolds CF 3rd, Kupfer DJ. Benzodiazepines and zolpidem for chronic insomnia: a meta-analysis of treatment efficacy. JAMA 1997;278:2170-7.

10. Hajak G. Insomnia in primary care. Sleep 2000;23 Suppl 3:S54-63.

11. Insomnia: assessment and management in primary care. National Heart, Lung, and Blood Institute Working Group on Insomnia. Am Fam Physician 1999;59:3029-38.

12. Ancoli-Israel S. Insomnia in the elderly: a review for the primary care practitioner. Sleep 2000;23:S23-30.

13. Ohayon MM, Caulet M, Priest RG, Guilleminault C. DSM-IV and ICSD-90 insomnia symptoms and sleep dissatisfaction. Br J Psychiatry 1997;171: 382-8.
14. Morin CM. Insomnia: psychological assessment and management. New York: Guilford Press; 1993.

15. American Psychiatric Association. Diagnostic and statistical manual of mental disorders. 4th ed. Washington DC: American Psychiatric Association; 2000.

16. Bastien CH, Vallières A, Morin CM. Validation of the insomnia severity index as an outcome measure for insomnia research. Sleep Med 2001;2:297-30.

17. Buysse DJ, Reynolds CF 3rd, Monk TH, Berman SR, Kupfer DJ. The Pittsburgh Sleep Quality Index: a new instrument for psychiatric practice and research. Psychiatry Res 1989;28:193-213.

18. Morin CM, Stone J, McDonald K, Jones S. Psychological management of insomnia: a clinical replication series with 100 patients. Behav Ther 1994;25: 291-309.

19. Johns MW. A new method for measuring daytime sleepiness: the Epworth sleepiness scale. Sleep 1991; 14:540-5.

20. Johns MW. Reliability and factor analysis of the Epworth Sleepiness Scale. Sleep 1992;15:376-81.

21. Fisk JD, Ritvo PG, Ross L, Haase DA, Marrie TJ, Schlech WF. Measuring the functional impact of fatigue: initial validation of the fatigue impact scale. Clin Infect Dis 1994;18 Suppl 1:S79-83.

22. Vanable PA, Aikens JE, Tadimeti L, Caruana-Montaldo B, Mendelson WB. Sleep time misperception among sleep disorder patients: variability as a function of diagnostic subgroupings and psychological characteristics [abstract]. Sleep 1998;21:S125. 